\title{
Upregulation of TREM2 Ameliorates Neuropathology and Rescues Spatial Cognitive Impairment in a Transgenic Mouse Model of Alzheimer's Disease
}

\author{
Teng Jiang', Lan Tan*,',2,3, Xi-Chen Zhu', Qiao-Quan Zhang ${ }^{4}$, Lei Cao', Meng-Shan Tan ${ }^{3}$, Li-Ze Gu', \\ Hui-Fu Wang', Zheng-Zheng Ding ${ }^{5}$, Ying-Dong Zhang ${ }^{6}$ and Jin-Tai Yu* ${ }^{*}, 1,2,3,7$ \\ 'Department of Neurology, Qingdao Municipal Hospital, Nanjing Medical University, Nanjing, People's Republic of China; ${ }^{2}$ Department of \\ Neurology, Qingdao Municipal Hospital, School of Medicine, Qingdao University, Qingdao, People's Republic of China; ' ${ }^{3}$ Department of Neurology, \\ Qingdao Municipal Hospital, College of Medicine and Pharmaceutics, Ocean University of China, Qingdao, People's Republic of China; \\ ${ }^{4}$ The Clinical Laboratory of Nanjing Brain Hospital, Nanjing Medical University, Nanjing, People's Republic of China; ${ }^{5}$ Department of Biochemistry \\ and Molecular Biology, Nanjing Medical University, Nanjing, People's Republic of China; ${ }^{6}$ Department of Neurology, Nanjing First Hospital, \\ Nanjing Medical University, Nanjing, People's Republic of China; ${ }^{7}$ Department of Neurology, Memory and Aging Center, University of California, \\ San Francisco, CA, USA
}

\begin{abstract}
Triggering receptor expressed on myeloid cells 2 (TREM2) gene is a recently identified susceptibility gene for Alzheimer's disease (AD), as its low-frequency variants increase the risk of this disease with an odds ratio similar to that of an APOE $\varepsilon 4$ allele. To date, the expression and biologic functions of TREM2 under AD context remain largely unknown. Using APPswe/PSI dE9 mice, a transgenic model of AD, we showed that TREM2 was upregulated in microglia during disease progression. For the first time, we provided in vitro and in vivo evidence that this upregulation was attributed to the increased amyloid- $\beta(A \beta)_{\mid}-42$ levels in the brain. By knockdown and overexpression of TREM2 in cultured primary microglia, we revealed that TREM2 modulated microglial functions under AD context, as it facilitated $A \beta_{1-42}$ phagocytosis and inhibited $A \beta_{1-42}$-triggered proinflammatory responses. Meanwhile, this modulation was dependent on DAPI2, the adapter protein of TREM2. More importantly, overexpression of TREM2 in the brain of APPswe/PSIdE9 mice markedly ameliorated AD-related neuropathology including $A \beta$ deposition, neuroinflammation, and neuronal and synaptic losses, which was accompanied by an improvement in spatial cognitive functions. Taken together, our data suggest that the upregulation of TREM 2 serves as a compensatory response to $A \beta_{1-42}$ and subsequently protects against $A D$ progression by modulation of microglia functions. These findings provide insights into the role of TREM2 in AD pathogenesis, and highlight TREM2 as a potential therapeutic target for this disease.

Neuropsychopharmacology (2014) 39, 2949-2962; doi:I0.1038/npp.2014.164; published online 20 August 20I4
\end{abstract}

\section{INTRODUCTION}

Recently, two genetic studies using next-generation sequencing have identified triggering receptor expressed on myeloid cells 2 (TREM2) gene as a novel susceptibility gene for Alzheimer's disease (AD), as a low-frequency variant within exon 2 of TREM2 increases the risk of this disease in Caucasians with an odds ratio similar to that of an APOE $\varepsilon 4$ allele (Guerreiro et al, 2013; Jonsson et al, 2013; Neumann and Daly, 2013). TREM2 is a 230-amino-acid type I transmembrane receptor that belongs to the immunoglobulin family (Daws et al, 2001). As its name suggests, TREM2 is expressed by a subset of myeloid cells including

*Correspondence: Dr JT Yu or Professor L Tan, Department of Neurology, Qingdao Municipal Hospital, School of Medicine, Qingdao University, No. 5 Donghai Middle Road, Qingdao, Shandong 26607I, People's Republic of China, Tel: +86 5328890 5659, Fax: +86532 8890 5659, E-mail: dr.tanlan@।63.com or yu-jintai@। 63.com Received 22 April 20 I4; revised 25 June 20।4; accepted 26 June 20।4; accepted article preview online 22 July 2014 monocytes, dendritic cells, osteoclasts, and tissue macrophages in peripheral tissues, coupling with DAP12 for its signaling and biologic functions (Colonna, 2003; Jiang et al, 2013). In the brain, TREM 2 was found to be expressed by the microglia (Frank et al, 2008; Jiang et al, 2014a; Neumann and Takahashi, 2007; Takahashi et al, 2005), the main immune cell within the central nervous system.

Under $\mathrm{AD}$ context, the microglia can be activated by amyloid- $\beta(\mathrm{A} \beta)$, the pathologic hallmark of $\mathrm{AD}$, and exerts a 'double-edged sword' function during the progression of this disease (Hensley, 2010; Meda et al, 2001; Wyss-Coray, 2006). On the one hand, activated microglia participates in the phagocytosis of $\mathrm{A} \beta$ through its phagocytic activity, and thus prevents the deposition of $\mathrm{A} \beta$ and the formation of amyloid plaques. On the other hand, long-term $\mathrm{A} \beta$ stimulation results in the dysfunction of microglia in the brain, which is characterized by the overproduction of proinflammatory cytokines, subsequently leading to the bystander neuronal and synaptic damages. Coincidentally, several lines of evidence have suggested an essential role of 
TREM2 in the modulation of microglial functions including phagocytosis and production of proinflammatory cytokines. In vitro, TREM2 could promote the phagocytosis of microsphere beads and apoptotic neurons by the microglia (Hsieh et al, 2009; Melchior et al, 2010; Takahashi et al, 2005). Meanwhile, TREM 2 was found to inhibit microgliamediated production of proinflammatory cytokines induced by lipopolysaccharides (LPSs) or apoptotic cells (Hsieh et al, 2009; Takahashi et al, 2005). In humans, TREM2 was revealed to have an essential role in the maintenance of the immune homeostasis in the brain via promotion of tissue debris clearance and resolution of latent inflammatory responses (Neumann and Takahashi, 2007), whereas mutation-induced dysfunction of TREM2 in microglia resulted in an autosomal recessive disorder called Nasu-Hakola disease, which is characterized by progressive presenile inflammatory neurodegeneration and severe cognitive impairment (Paloneva et al, 2002). Despite the above information, the expression and biologic functions of TREM2 in the brain under AD context remain largely unknown. Whether the expression of TREM2 is dysregulated in the $\mathrm{AD}$ brain remains unclear. Furthermore, whether TREM2 has an essential role in the modulation of microglial functions during $\mathrm{AD}$ progression and whether TREM2 represents a potential therapeutic target for this disease still remains unexplored.

Herein, using APPswe/PS1dE9 mice, a transgenic model of $\mathrm{AD}$, we showed that TREM2 was upregulated in microglia under $\mathrm{AD}$ context. Subsequent in vitro and in vivo experiments showed that this upregulation was attributed to the increase in $\mathrm{A} \beta_{1-42}$ levels. By knockdown and overexpression of TREM2 in primary microglia, we revealed that TREM2 modulated microglial functions under $\mathrm{AD}$ context, as it facilitated $\mathrm{A} \beta_{1-42}$ phagocytosis and inhibited $\mathrm{A} \beta_{1-42^{-}}$ induced proinflammatory response. Meanwhile, this modulation was dependent on DAP12, the adapter protein of TREM2. More importantly, TREM2 overexpression in the brain of APPswe/PS1dE9 mice ameliorated AD-related neuropathology including $\mathrm{A} \beta$ deposition, neuroinflammation, and neuronal and synaptic losses, which was accompanied by an improvement in spatial cognitive functions. These data indicate that the upregulation of TREM2 serves as a compensatory response to $A \beta_{1-42}$ and subsequently protects against $\mathrm{AD}$ progression by modulation of microglia functions. These findings provide insights into the role of TREM2 in AD pathogenesis, and highlight TREM2 as a potential therapeutic target for this disease.

\section{MATERIALS AND METHODS}

\section{Animals}

Male APPswe/PS1dE9 (expressing Mo/HuAPP695 and PS1dE9 on the C57Bl/6 background) transgenic mice and their age-matched wild-type (WT) mice were purchased from the Jackson Laboratory. Animal Care and Management Committee of Qingdao Municipal Hospital approved the whole study protocol. All experiments were conducted in accordance with Guide for the Care and Use of Laboratory Animals of the National Institutes of Health.

\section{Isolation of Primary Microglia}

Microglia was isolated from the brain of WT mice at postnatal day 1 as described by Griciuc et al (2013). The purity of the isolated microglia was determined by immunostaining with antibody against Iba- 1 . On average, over $90 \%$ of cultured cells were immunostained with Iba-1.

\section{In vitro $\mathrm{A} \beta$ stimulation and intracerebral $\mathrm{A} \beta$ injection}

The oligomeric form of $\mathrm{A} \beta_{1-42}$ was prepared according to a published protocol (Larson and Lesne, 2012).

For in vitro $\mathrm{A} \beta$ stimulation, primary microglia was seeded at a density of $2 \times 10^{5}$ cells per ml into 24 -well plates, and was stimulated with vehicle, $\mathrm{A} \beta_{1-42}$, or $\mathrm{A} \beta_{42-1}$ for $24 \mathrm{~h}$. Cells were collected for quantitative real-time polymerase chain reaction (qRT-PCR) and western blot analysis.

Intracerebral $\mathrm{A} \beta$ injection was conducted by surgeons who were unaware of the experimental groups. Seven-monthold WT mice were anesthetized $(80 \mathrm{mg} / \mathrm{kg}$ ketamine hydrochloride, $5 \mathrm{mg} / \mathrm{kg}$ xylazine hydrochloride) and fixed on a stereotactic frame. $\mathrm{A} \beta_{1-42}$ or $\mathrm{A} \beta_{42-1}$ was injected into the cerebral cortex (anteroposterior: - 2; mediolateral: 1.2; dorsoventral: $-1.2 \mathrm{~mm}$ ) and the hippocampus (anteroposterior: -2 ; mediolateral: 1.2 ; dorsoventral: $-2 \mathrm{~mm}$ ) of the right hemisphere by using a micropipette attached to a $10 \mu \mathrm{l}$ Hamilton syringe. Meanwhile, $2 \mu \mathrm{l}$ artificial cerebral spinal fluid (aCSF) was used as a control solution. Twenty-four hours later, mice were killed. The cerebral cortex and hippocampus were used for qRT-PCR and western blot analysis.

\section{Lentiviral Vector Preparation}

Lentiviral vectors encoding the mouse TREM2 gene (NCBI ID: NM_031254.3) and a control lentiviral vector were provided by Keygen Biotech. Co. (Nanjing). Meanwhile, the following short hairpin sequences (TREM2 shRNA: $5^{\prime}$-TGA TGCTGGAGATCTCTGGGTTCAAGAGACCCAGAGATCTC CAGCATCTTTTTTC- $3^{\prime}$ and control shRNA: 5'-TGATGCT GAAGGTCGCTTGGTTCAAGAGACCAAGCGACCTCCAGC ATCTTTTTTC- $3^{\prime}$ ) were synthesized and cloned into the lentiviral vectors, respectively. The lentiviral vectors were purified and then co-transfected with packaging vectors (Invitrogen) into 293FT cells (Invitrogen). The supernatant was collected after $48 \mathrm{~h}$, and the lentiviral particles in the supernatant were concentrated at 1:100 by ultracentrifugation and recovered by suspension in PBS. For knockdown of DAP12, the lentiviral particles containing DAP12 shRNA and control shRNA were purchased from Santa Cruz and directly recovered by suspension in PBS. The titers of lentiviral particles ranged between $10^{6}$ and $10^{7}$ multiplicity of infection.

\section{Primary Microglia Transduction and Intracerebral Lentiviral Particle Injection}

Purified microglia was seeded at a density of $2 \times 10^{5}$ cells per $\mathrm{ml}$ into 24 -well plates. Lentiviral particles and $8 \mu \mathrm{g} / \mathrm{ml}$ polybrene (Sigma-Aldrich) were added to the culture and centrifuged for $90 \mathrm{~min}$ at 1500 r.p.m. The supernatant was removed immediately after infection and replaced with basal medium (Invitrogen) containing 10\% fetal calf 
serum and 50\% glial culture supernatant obtained from the culture before transduction. The efficiency of transduction was determined by qRT-PCR and western blot analysis $72 \mathrm{~h}$ later.

Stereotactic intracerebral injection of lentiviral particles was performed by surgeons who were unaware of the experimental groups. Seven-month-old APPswe/PS1dE9 mice and their age-matched WT mice were anesthetized and fixed on a stereotactic frame. Injection of lentiviral particles was performed in the cerebral cortex (two deposits) and hippocampus (one deposit) of each hemisphere with $2 \mu \mathrm{l}$ lentiviral preparation using a micropipette attached to a $10 \mu \mathrm{l}$ Hamilton syringe. Stereotactic coordinates of injection sites from bregma were (1) anteroposterior: -0.3 , mediolateral: 2 , dorsoventral: $-1.5 \mathrm{~mm}$ and anteroposterior: -2 , mediolateral: 1.2, dorsoventral: $-1.2 \mathrm{~mm}$ for the cerebral cortex; and (2) anteroposterior: -2 ; mediolateral: 1.2 ; dorsoventral: $-2 \mathrm{~mm}$ for the hippocampus. Two months later, the therapeutic effects of lentiviral vector-mediated TREM2 overexpression were verified. According to one of our preliminary experiments, the expression of TREM2 in the brain of APPswe/PS1dE9 mice reached a relatively steady level at 1 month after the lentiviral particle injection (data not shown). Meanwhile, as reported by Dodart et al (2005), obvious neuronal losses were observed in the brain at 3 months after lentiviral vector-mediated gene delivery, possibly owing to the longterm neurotoxicity of lentivirus. On consideration of these factors, '2 months' was selected as the time point for investigation.

\section{qRT-PCR}

Total RNA in cultured primary microglia and brain tissues was extracted by Trizol reagent (Invitrogen). Equal amounts of total RNA was reverse transcribed under standard conditions using the PrimeScript RT Master Mix (Takara). After that, qRT-PCR reactions were performed with SYBR Premix Ex Taq (Takara) and specific mouse primers (see Supplementary Table S1 for detail) according to the manufacturer's instructions. GAPDH was adopted as an internal control.

\section{Western Blot Analysis}

Cultured primary microglia and brain tissues were lysed in an extraction buffer. Different samples with an equal amount of protein were separated on SDS-polyacrylamide gels, transferred to PVDF membranes, and blocked with 5\% bovine serum albumin. Membranes were incubated overnight with the primary antibody against TREM2 (Santa Cruz), DAP12 (Santa Cruz), Iba-1 (Abcam), synaptophysin (Abcam) or C-terminal of APP (Sigma-Aldrich), and then washed again and incubated with HRP-coupled secondary antibody. Protein bands were detected with chemiluminescent HRP substrate (Thermo Scientific). The signal intensity of primary antibody binding was analyzed using the Quantity One software 4.6.2 (Bio-Rad Laboratories).

\section{ELISA}

Brain tissues were homogenized in Tris-buffered saline containing $5 \mathrm{mM}$ EDTA, phosphatase inhibitor, protease inhibitor cocktail, and $2 \mathrm{mM} 1,10$-phenanthroline. The homogenate was centrifuged at $100000 \mathrm{~g}$ for $1 \mathrm{~h}$. Supernatants were collected and used to measure soluble forms of $\mathrm{A} \beta$. The resulting pellet was homogenized in $70 \%$ formic acid (FA). Samples were centrifuged at $100000 \mathrm{~g}$ for $1 \mathrm{~h}$ and supernatants were collected. FA-containing supernatants were neutralized with $1 \mathrm{M}$ Tris base and samples were used to measure insoluble forms of $\mathrm{A} \beta$. $\mathrm{A} \beta_{1-40}$ and $\mathrm{A} \beta_{1-42}$ levels were measured using A $\beta$ ELISA Kits (Invitrogen) according to the manufacturer's protocol.

\section{A $\beta$ Phagocytosis and Degradation Assays}

An in vitro assay described by Griciuc et al (2013)was used to determine microglial $\mathrm{A} \beta_{1-42}$ phagocytosis and degradation. Primary mouse microglia was treated with $0.5 \mu \mathrm{M}$ $\mathrm{A} \beta_{1-42}$ for $6 \mathrm{~h}$. According to our preliminary experiment, stimulation with $0.5 \mu \mathrm{M}$ of $\mathrm{A} \beta_{1-42}$ for $6 \mathrm{~h}$ did not induce upregulation of TREM2 in primary microglia. After incubation, cells were then washed with DMEM and maintained for additional $6 \mathrm{~h}$ in serum- and $\mathrm{A} \beta_{1-42}$-free DMEM. Later, cells were washed again and were lysed in cell lysis buffer supplemented with protease inhibitors (Roche) and $2 \mathrm{mM}$ 1,10 phenanthroline (Sigma). Lysates were centrifuged at $12000 \mathrm{~g}$ for $15 \mathrm{~min}$. Supernatants were collected for $\mathrm{A} \beta_{1-42}$ ELISA.

\section{Histologic Analysis}

Brain section for histologic analysis was prepared as described (Jiang et al, 2014a). Nissl staining was performed as described (Tan et al, 2014). Three coronal sections at different depths on the rostrocaudal axis $(-0.8 \mathrm{~mm}$, $-1.8 \mathrm{~mm}$, and $-2.8 \mathrm{~mm}$ from bregma) were imaged for each animal. Neurons with dark violet nucleus and intact morphology were identified as Nissl-positive neurons. Six fields of cortex and hippocampus on each coronal section were then randomly selected, and the numbers of Nisslpositive neurons were counted by observers who were unaware of the experimental groups.

For double immunofluorescence staining, sections were incubated overnight with an antibody against TREM2 (Santa Cruz) combined with an antibody against Iba-1 (Abcam), an antibody against glial fibrillary acidic protein (GFAP; Abcam), an antibody against neuron-specific nuclear protein (NeuN; Abcam), or an antibody against oligodendrocyte-specific protein (OSP; Santa Cruz). Sections were then washed and sequentially incubated with FITC-conjugated anti-rabbit IgG (Zhongshan Golden Bridge) and TRITC-conjugated anti-mouse IgG (Zhongshan Golden Bridge). Then, the sections were washed again and sealed with a coverslip. The slides were then analyzed with a fluorescence microscope.

For immunohistochemistry analysis, the sections were incubated with a rabbit polyclonal antibody against TREM2 (Santa Cruz) or a mouse monoclonal antibody against A $\beta$ (4G8, which recognizes amino-acid residues 17-24 of $\mathrm{A} \beta$; Covance), and then treated with biotinylated goat 
anti-rabbit IgG or goat anti-mouse IgG (Zhongshan Golden Bridge). Immunoreactivity was detected with diaminobenzidine. Last, sections were dehydrated, mounted on the slides, and examined with a light microscope. Assessment of amyloid plaque density and total amyloid burden was conducted in a blind manner as described by MorenoGonzalez et al (2013).

\section{Morris Water Maze Test}

Morris water maze test was performed in a blind manner at the last 6 days before mice were killed (Jiang et al, 2014b). Mice were given four training trials per day for 5 consecutive days. The path length to the submerged platform was recorded, and the average time of four trials was calculated. Twenty-four hours after the last trial, mice were subjected to a probe test in which the platform was removed, and their swimming paths were recorded.

\section{Statistical Analysis}

Independent sample $t$-test or one-way ANOVA followed by Tukey's post hoc test were used to analyze differences among groups. Pearson's correlation analysis was used to investigate the correlation between the expressions of $\mathrm{A} \beta$ and TREM2. For the hidden-platform training of the Morris water maze test, the path length was analyzed by two-way repeated-measures ANOVA followed by Tukey's post hoc test. Data are expressed as mean \pm SEM. $P<0.05$ was considered statistically significant.

\section{RESULTS}

\section{TREM2 is Localized on Microglia and is Upregulated under AD Context}

First, we investigated the cellular localization of TREM2 in the brain of 7-month-old APPswe/PS1dE9 mice. According to our previous findings, AD-related pathophysiologic changes, including amyloid plaque formation, neuroinflammation, and spatial cognitive impairment were apparent on APPswe/PS1dE9 mice at this age (Jiang et al, 2014b). As indicated in Figure 1a, double immunofluorescence staining indicated a good colocalization of TREM2 and microglial marker Iba-1 in the brain. Meanwhile, we found that TREM2 was expressed by microglia in both plaque-associated and plaque-free regions (Supplementary Figure S1). However, no expression of TREM2 was detected on NeuN-labeled neurons, GFAP-labeled astrocytes, or OSP-labeled oligodendrocytes.

Next, we detected the levels of TREM2 and its adapter protein DAP12 in the brains of 7-month-old APPswe/ PS1dE9 mice and their age-matched WT mice. A 2.4-fold increase in TREM2 mRNA level was observed in the cerebral cortex of APPswe/PS1dE9 mice relative to WT mice $(n=8, t=11.96, P<0.05$; Figure $1 \mathrm{~b})$. Meanwhile, the mRNA level of TREM2 in the hippocampus of APPswe/ PS1dE9 mice was threefold higher than those of WT mice ( $n=8, t=14.23, P<0.05$; Figure $1 \mathrm{~g}$ ). Later, we investigated whether TREM2 protein levels showed a corresponding increase in the brain of 7-month-old APPswe/PS1dE9 mice. As shown in Figure 1d and e, a 1.7-fold increase in TREM2 protein level in the cerebral cortex of APPswe/PS1dE9 mice relative to WT mice was observed $(n=8, t=5.9, P<0.05)$. Meanwhile, the protein level of TREM2 in the hippocampus of APPswe/PS1dE9 mice was 2.2-fold higher than those of WT mice (Figure $1 \mathrm{i}$ and $\mathrm{j}, n=8, t=11.12, P<0.05$ ). These differences remained statistically significant when normalizing TREM2 levels to the expression of Iba-1(Figure $1 \mathrm{f}$ and $\mathrm{k}$ ), implying that the upregulation of TREM2 was not attributed to an increase in the number of microglia in AD. In addition, no significant difference was observed in DAP12 expression in the brains of 7-month-old APPswe/PS1dE9 mice and their age-matched WT mice (Figure 1c and h). Collectively, these findings suggest that TREM2 was upregulated in microglia under $\mathrm{AD}$ context.

TREM2 Upregulation in Microglia is a Direct Response to the Increase in $A \beta_{1-42}$ Levels

Next, we investigated the relation between the expression of TREM 2 and $\mathrm{A} \beta$, the pathologic hallmark of $\mathrm{AD}$, in the brain of 7-month-old APPswe/PS1dE9 mice. As indicated in Figure $2 \mathrm{a}$ and $\mathrm{b}$, positive correlations were observed between the expression of TREM2 and the levels of soluble $(n=8, r=0.673, P=0.033)$ and insoluble $(n=8, r=0.751$, $P=0.012)$ forms of $\mathrm{A} \beta_{1-42}$, the most toxic $\mathrm{A} \beta$ species in $\mathrm{AD}$ pathogenesis. However, no correlation was found between the expression of TREM2 and the levels of soluble $(n=8$, $r=0.508$, NS) and insoluble $(n=8, r=0.611$, NS) forms of $\mathrm{A} \beta_{1-40}$ in the brain of APPswe/PS1dE9 mice (Figure $2 \mathrm{c}$ and $\mathrm{d}$ ). Based on these findings, we proposed that TREM2 upregulation in microglia might be attributed to the increase in $\mathrm{A} \beta_{1-42}$ levels under $\mathrm{AD}$ context.

To test this hypothesis, cultured primary microglia was challenged with vehicle or 5,10 , or $25 \mu \mathrm{M}$ of $\mathrm{A} \beta_{1-42}$ for $24 \mathrm{~h}$, and the mRNA and protein levels of TREM2 were investigated by qRT-PCR and western blot analysis, respectively. As shown in Figures $2 \mathrm{e}-\mathrm{g}, \mathrm{A} \beta 1_{-42}$ significantly increased the mRNA $\left(\mathrm{F}_{(4,25)}=140.5, P<0.05\right)$ as well as the protein levels $\left(\mathrm{F}_{(4,25)}=35.76, P<0.05\right)$ of TREM2 in a dosedependent manner. To evaluate the specificity of this test, primary microglia was incubated with $25 \mu \mathrm{M}$ of reverse $\mathrm{A} \beta_{42-1}$ for $24 \mathrm{~h}$. As expected, reverse $\mathrm{A} \beta_{42-1}$ peptide was unable to increase the mRNA $(q=0.34$, NS) and protein levels $(q=0.42$, NS) of TREM2 in the primary microglia (Figure $2 \mathrm{e}-\mathrm{g}$ ). To validate the above findings in vivo, different doses of $\mathrm{A} \beta_{1-42}\left(1,2\right.$, or $5 \mu \mathrm{g}$ of $\mathrm{A} \beta_{1-42}$ dissolved in $2 \mu \mathrm{l}$ aCSF) was stereotaxically injected into the cortex and hippocampus of 7-month-old WT mice. At $24 \mathrm{~h}$ after injection, the mRNA and protein levels of TREM2 in the brain were investigated by qRT-PCR and western blot analysis, respectively. As revealed by Figure $2 \mathrm{~h}-\mathrm{m}, \mathrm{A} \beta 1_{-42}$ elevated the mRNA and protein levels of TREM2 dosedependently in both the cortex $(n=8$; TREM2 mRNA levels: $\mathrm{F}_{(4,35)}=106, P<0.05$; TREM2 protein levels: $\mathrm{F}_{(4,35)}=46.31$, $P<0.05)$ and hippocampus $(n=8$; TREM2 mRNA levels: $\mathrm{F}_{(4,35)}=46.92, \quad P<0.05$; $\quad$ TREM2 protein levels: $\left.\mathrm{F}_{(4,35)}=36.93, P<0.05\right)$ of WT mice. It is worth noting that injection of reverse $\mathrm{A} \beta_{42-1}\left(5 \mu \mathrm{g}\right.$ of $\mathrm{A} \beta_{42-1}$ dissolved in $2 \mu \mathrm{l}$ aCSF) did not affect the mRNA and protein levels of TREM2 in the cortex $(n=8$; TREM2 mRNA levels: $q=0.29$, NS; TREM2 protein levels: $q=0.16$, NS) and hippocampus $(n=8$; TREM2 mRNA levels: $q=0.13$, NS; TREM2 protein 

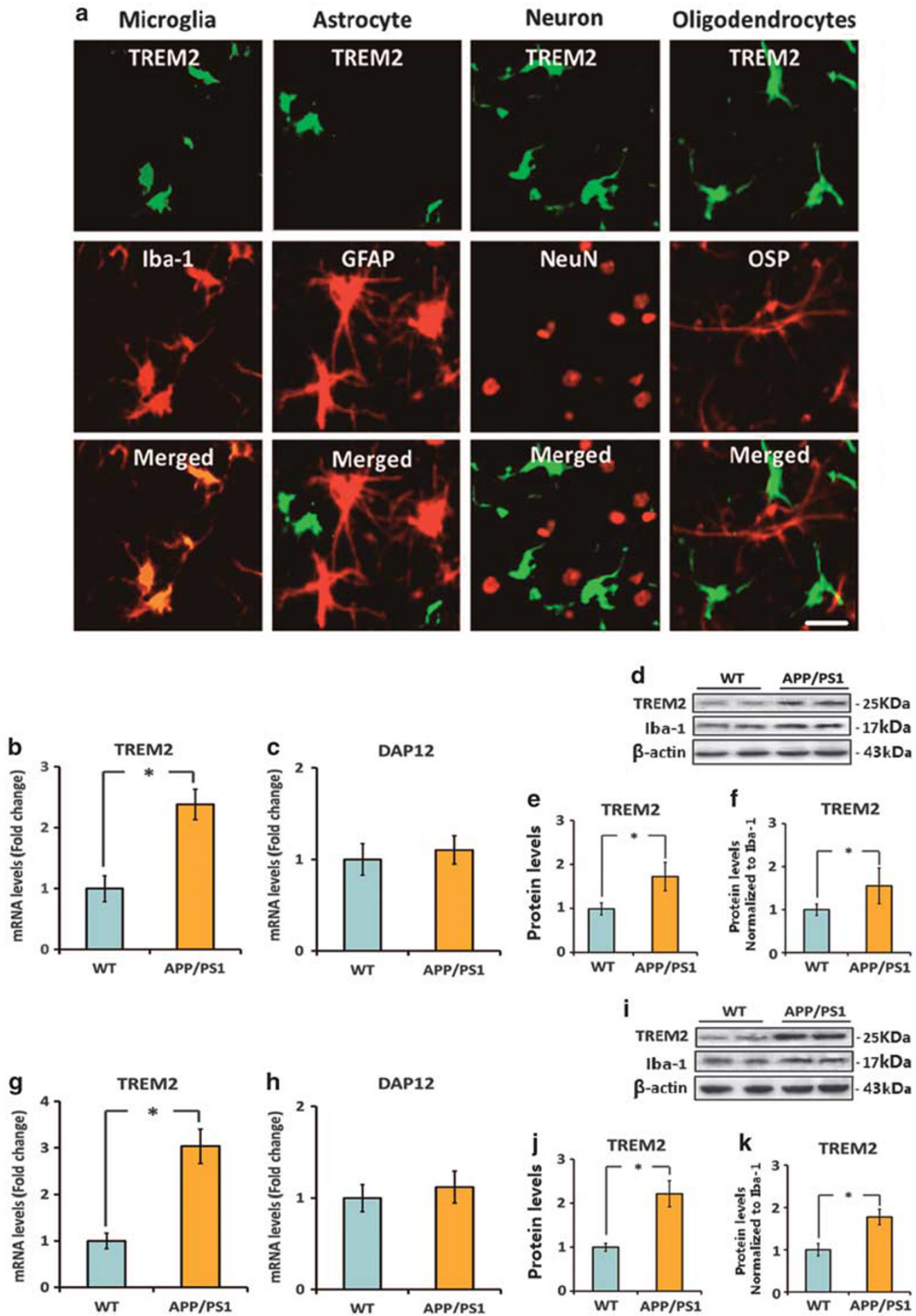

Figure I Triggering receptor expressed on myeloid cells 2 (TREM2) is localized on microglia and is upregulated under Alzheimer's disease (AD) context. (a) The cellular localization of TREM2 in the brain of 7-month-old APPswe/PSI dE9 mice was investigated by double immunofluorescence staining. TREM2 was colocalized with Iba-I (a microglia marker) rather than GFAP (an astrocyte marker), NeuN (a neuron marker), and OSP (an oligodendrocyte marker). Scale bar $=20 \mu \mathrm{m}$. (b and c) mRNA levels of TREM2 and DAPI2 in the cerebral cortex of 7-month-old APPswe/PS I dE9 mice and wild-type (WT) mice were measured by quantitative reverse transcription-PCR (qRT-PCR). Data were normalized to the levels of GAPDH mRNA. ( $d-f$ ) Protein levels of TREM2 in the cerebral cortex of 7-month-old APPswe/PSI dE9 mice and WT mice were detected by western blot analysis. Data were normalized to $\beta$-actin or a microglia marker lba-l. (g and h) mRNA levels of TREM2 and DAPI2 in the hippocampus of 7-month-old APPswe/PSIdE9 mice and WT mice were measured by qRT-PCR. Data were normalized to the levels of glyceraldehyde 3-phosphate dehydrogenase (GAPDH) mRNA. (i-k) Protein levels of TREM2 in the hippocampus of 7-month-old APPswe/PSI dE9 mice and WT mice were detected by western blot analysis. Data were normalized to $\beta$-actin or a microglia marker Iba-I. All data were analyzed by independent sample $t$-test. Columns represent mean \pm SEM ( $n=8$ per group). *P $<0.05$. It should be noted that cropped gels are used in this figure, and the full-length gels are shown in Supplementary Figure S6. 
a

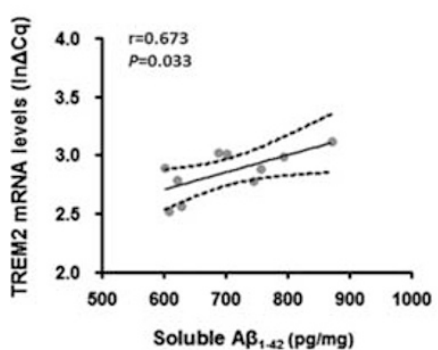

C
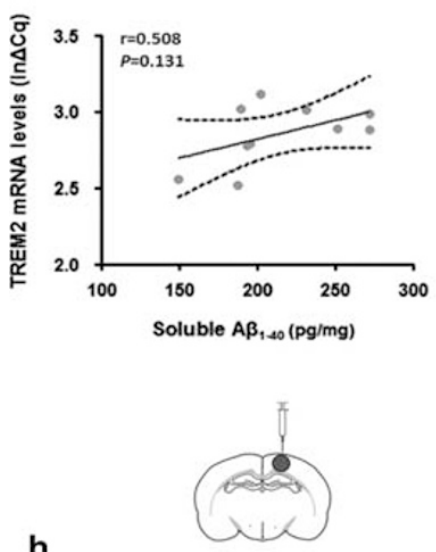

h

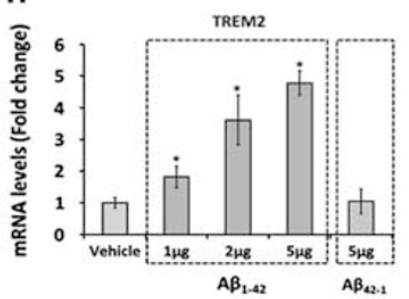

j

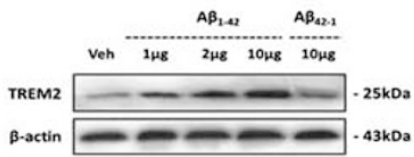

b

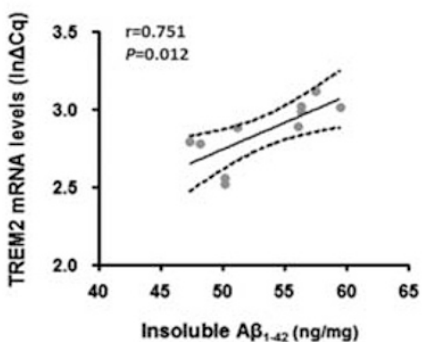

d

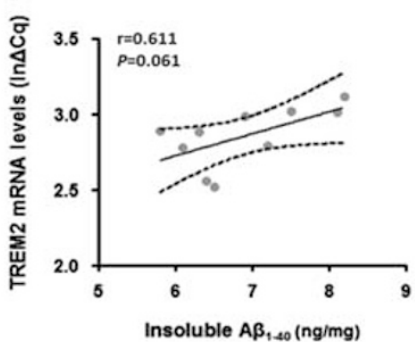

Primary microglia

e

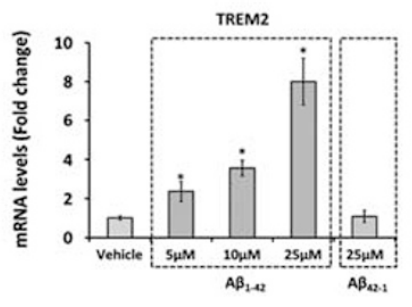

g

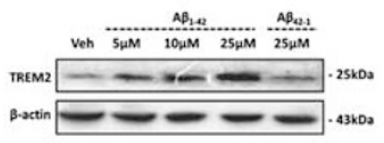

f
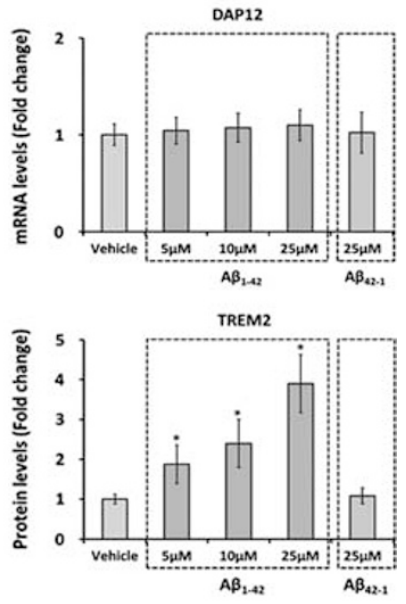

\section{Cerebral cortex}

i
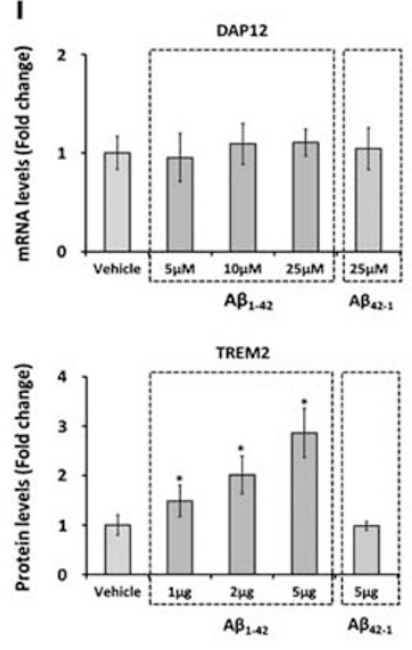

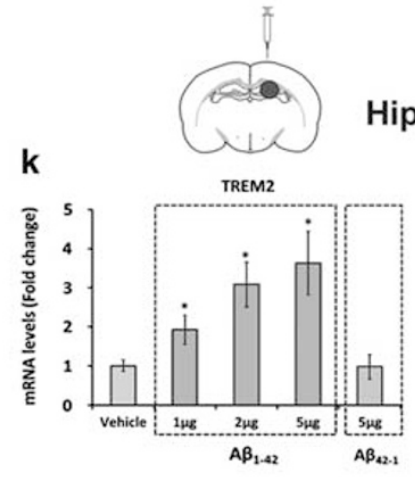

Hippocampus

m

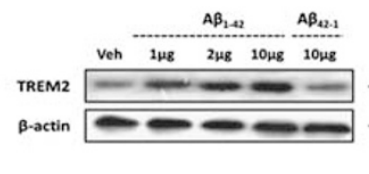

I
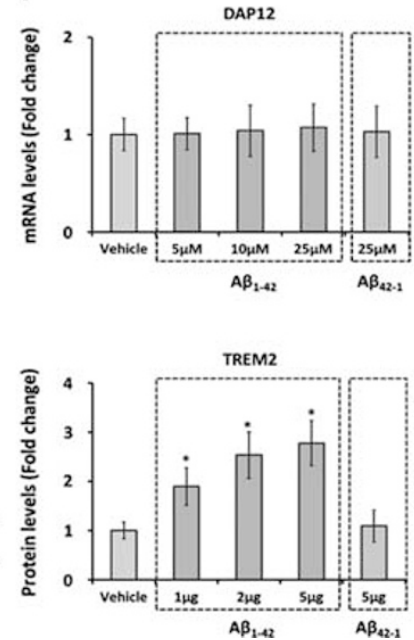

Figure 2 Triggering receptor expressed on myeloid cells 2 (TREM2) upregulation in microglia is a direct response to the increase of amyloid- $\beta(A)$ । -42 levels. The levels of soluble and insoluble forms of $A \beta_{1-42}$ and $A \beta_{1-40}$ were detected by enzyme-linked immunoassay (ELISA), and Pearson's correlation analysis showed that TREM2 expression is positively correlated with soluble and insoluble forms of $A \beta_{\perp-42}$ (a and b) rather than soluble and insoluble forms of $A \beta_{1-40}$ (c and d). (e-g) Cultured primary microglia was incubated with vehicle, $A \beta_{42-1}$, or different doses of $A \beta_{1-42}$ for $24 \mathrm{~h}$. Later, the TREM2 and DAPI2 mRNA levels and the protein levels of TREM2 within cells were measured by quantitative reverse transcription-PCR (qRT-PCR) and western blot, respectively. (h-j) Vehicle, $A \beta_{42-1}$, or different doses of $A \beta_{1-42}$ was injected into the cerebral cortex of the right hemisphere of 7-month-old WT mice. At $24 \mathrm{~h}$ after injection, the TREM2 and DAPI2 mRNA levels and the protein levels of TREM 2 in the cerebral cortex of the right hemisphere were measured by qRT-PCR and western blot analysis, respectively ( $n=8$ per group). ( $k-m)$ Vehicle, $A \beta_{42-1}$, or different doses of $A \beta_{1-42}$ was injected into the hippocampus of the right hemisphere of 7-month-old $W T$ mice. Twenty-four hours later, the TREM2 and DAPI 2 mRNA levels and the protein levels of TREM2 in the hippocampus of the right hemisphere were measured by qRT-PCR and western blot analysis, respectively ( $n=8$ per group). The data of qRT-PCR were normalized to the levels of glyceraldehyde 3-phosphate dehydrogenase (GAPDH) mRNA, and the data of western blot analysis were normalized to $\beta$-actin. Data were analyzed by Pearson's correlation analysis (a-d) or one-way analysis of variance (ANOVA) followed by Tukey's post hoc test $(\mathrm{e}-\mathrm{m})$. Columns represent mean $\pm \mathrm{SEM}$. * $P<0.05$ vs vehicle-treated cells or animals. It should be noted that cropped gels are used in this figure, and the full-length gels are shown in Supplementary Figure S6.

levels: $q=0.68$, NS) of WT mice (Figure $2 \mathrm{~h}-\mathrm{m}$ ). In addition, $\mathrm{A} \beta_{1-42}$ stimulation did not affect DAP12 expression in the primary microglia or in the brain of WT mice (Figure 2f, i and 1). Taken together, these findings indicated that the upregulation of TREM2 in microglia was a direct response to the increase in $\mathrm{A} \beta_{1-42}$ levels under $\mathrm{AD}$ context. 
TREM2 Facilitates Microglial Phagocytosis of $A \beta_{1-42}$ in a DAP12-Dependent Manner

We used a lentiviral strategy to manipulate TREM2 and DAP12 expression in the primary microglia, and the alterations in TREM2 and DAP12 levels were determined by qRT-PCR and western blot analysis $72 \mathrm{~h}$ later. As shown in Supplementary Figure S2, lentiviral particles containing TREM2 shRNA significantly reduced TREM2 mRNA and protein levels by $85 \%$ and $78 \%$, respectively. Meanwhile, lentiviral vector-mediated overexpression strategy increased the TREM2 mRNA and protein levels by 5.4 - and 3.8 -fold, respectively (Supplementary Figure S2). In addition, the DAP12 mRNA and protein levels in the primary microglia were reduced by $84 \%$ and $82 \%$, respectively, after infection with lentiviral particles containing DAP12 shRNA (Supplementary Figure S3). Later, an in vitro assay described by Griciuc et al (2013) was used to determine microglial $\mathrm{A} \beta_{1-42}$ phagocytosis and degradation: cells were incubated with $\mathrm{A} \beta_{1-42}$ for $6 \mathrm{~h}$, and the intracellular $\mathrm{A} \beta_{1-42}$ levels were detected by ELISA for the assessment of microglial $\mathrm{A} \beta_{1-42}$ phagocytosis. Next, the $\mathrm{A} \beta_{1-42}$ left in the medium was washed out, and the cells were maintained for an additional $6 \mathrm{~h}$ to allow for $\mathrm{A} \beta_{1-42}$ degradation. As shown in Figure 3a, knockdown of TREM2 resulted in a 51\% reduction in internalized $\mathrm{A} \beta_{1-42}$ levels in the primary microglia $(q=7.08$, $P<0.05)$. Meanwhile, knockdown of DAP12 resulted in a $52 \%$ reduction in internalized $\mathrm{A} \beta_{1-42}$ levels in the primary microglia $(q=6.98, P<0.05)$. However, overexpression of TREM2 resulted in a remarkable increase of $64 \%$ in internalized $\mathrm{A} \beta_{1-42}$ levels $(q=8.75, P<0.05)$. In addition, DAP12 knockdown fully abolished the increase in internalized $\mathrm{A} \beta_{1-42}$ levels induced by TREM2 overexpression $(q=11.93, P<0.05)$. It should be noted that manipulation of TREM2 or DAP12 expression did not significantly influence $\mathrm{A} \beta_{1-42}$ degradation rate in the primary microglia (Figure $3 \mathrm{~b}$ and c). Collectively, these findings suggested that TREM2 facilitated phagocytosis of $\mathrm{A} \beta_{1-42}$ in the primary microglia in a DAP12-dependent manner.

\section{TREM2 Directly Inhibits $A \beta_{1-42}$-Induced Proinflammatory Response in the Primary Microglia in a DAP12-Dependent Manner}

Next, the primary microglia was stimulated with $5 \mu \mathrm{M}$ of $\mathrm{A} \beta_{1-42}$ or $\mathrm{A} \beta_{42-1}$ for $3 \mathrm{~h}$ under serum-free conditions, and the mRNA levels of proinflammatory cytokines including tumor necrosis factor (TNF)- $\alpha$, interleukin (IL)-1 $\beta$, IL-6, and inducible nitric oxide synthase (iNOS) were determined by qRT-PCR. It is should be noted that stimulation with $5 \mu \mathrm{M}$ of $\mathrm{A} \beta_{1-42}$ for $3 \mathrm{~h}$ did not induce the upregulation of TREM2 in the primary microglia (data not shown). As shown in Figure 3d-g, A $\beta 1_{-42}$ significantly increased the expression of TNF- $\alpha$ (1.7 \pm 0.2 -fold, $n=8, \quad q=4.87$, $P<0.05)$, IL-1 $\beta \quad(2.2 \pm 0.3$-fold, $n=8, q=7.37, P<0.05)$, IL-6 (3.5 \pm 0.5 -fold, $n=8, q=9.13, P<0.05)$, and iNOS (1.9 \pm 0.2 -fold, $n=8, q=5.89, P<0.05)$. Meanwhile, $\mathrm{A} \beta_{42-1}$ did not affect the expression of these proinflammatory cytokines in the primary microglia, confirming the specificity of $\mathrm{A} \beta_{1-42}$ in induction of proinflammatory response (Figure $3 \mathrm{~d}-\mathrm{g}$ ). TREM2 knockdown markedly elevated the expression of TNF- $\alpha$, IL- $1 \beta$, IL- 6 , and iNOS by $47 \%(n=8$, $q=5.63, P<0.05), 96 \%(n=8, q=12.23, P<0.05), 46 \%$ $(n=8, q=5.94, \quad P<0.05)$, and $51 \% \quad(n=8, q=6.83$, $P<0.05)$, respectively, in primary microglia received $A \beta_{1-}$ ${ }_{42}$ stimulation (Figure $3 \mathrm{~d}-\mathrm{g}$ ). Meanwhile, knockdown of DAP12 also resulted in a significant increase in the expression of TNF- $\alpha$, IL- $1 \beta$, IL- 6 , and iNOS by $52 \%$ $(n=8, \quad q=6.18, \quad P<0.05), \quad 119 \% \quad(n=8, \quad q=14.48$, $P<0.05), 72 \%(n=8, q=9.14, P<0.05)$, and $67 \%(n=8$, $q=8.63, P<0.05)$, respectively. Overexpression of TREM2 remarkably attenuated the $\mathrm{A} \beta_{1-42}$-induced increase in TNF$\alpha$, IL- $1 \beta$, IL- 6 , and iNOS by $41 \%(n=8, q=4.89, P<0.05)$, $46 \% \quad(n=8, \quad q=6.19, \quad P<0.05), \quad 61 \% \quad(n=8, \quad q=7.4$, $P<0.05)$, and $39 \%(n=8, q=5.03, P<0.05)$, respectively, in the primary microglia (Figure $3 \mathrm{~d}-\mathrm{g}$ ). In addition, knockdown of DAP12 completely abrogated the effects of TREM2 overexpression on the expression of TNF- $\alpha(n=8$, $q=5.8, P<0.05)$, IL-1 $\beta(n=8, q=10.74, P<0.05)$, IL-6 $(n=8, q=11.03, \quad P<0.05)$, and iNOS $(n=8, q=6.2$, $P<0.05)$. As shown above, manipulation of TREM2 expression in microglia could affect its capacity to uptake $\mathrm{A} \beta_{1-42}$. Hence, it is possible that this will subsequently influence $\mathrm{A} \beta_{1-42}$ concentration in the culture medium and leads to the alteration in the production of proinflammatory cytokines. However, the concentration of $\mathrm{A} \beta_{1-42}$ in the culture medium was not varied among groups at the end of the experiment (data not shown). All these findings suggested that TREM2 directly inhibited $\mathrm{A} \beta_{1-42}$-induced proinflammatory response in the primary microglia in a DAP12-dependent manner.

\section{TREM2 Overexpression Ameliorates AD-Related Neuropathology in APPswe/PS1dE9 Mice}

To further validate the protective role of TREM2 under AD context in vivo, we used a lentiviral strategy to overexpress TREM2 in the brain of APPswe/PS1dE9 mice. As shown in Supplementary Figure S4, lentiviral preparations were injected bilaterally into the cortex and hippocampus of 7-month-old APPswe/PS1dE9 mice, and the increase in the expression of TREM2 in the brain was confirmed by RTPCR, western blot, and immunohistochemistry analyses 2 months later. First, we determined the effects of TREM2 overexpression on $\mathrm{A} \beta$ deposition by immunohistochemistry analysis and ELISA. As indicated in Figure 4a and b, TREM2 overexpression significantly attenuated amyloid plaque density in the cortex and hippocampus of APPswe/ PS1dE9 mice, as plaque numbers in these two regions were remarkably reduced by $56 \%(n=8, q=9.56, P<0.05)$ and $35 \%(n=8, q=5.2, P<0.05)$, respectively. In addition, the total amyloid burden in the cortex and hippocampus was also decreased by $66 \%(n=8, q=11.94, P<0.05)$ and $74 \%$ $(n=8, q=9.49, P<0.05)$, respectively, after TREM2 overexpression (Figure 4a and c). Meanwhile, overexpression of TREM2 markedly decreased soluble and insoluble forms of $\mathrm{A} \beta_{1-42}$ by $42 \%(n=8, q=6.82, P<0.05)$ and $34 \%(n=8$, $q=9.32, P<0.05$ ), respectively (Figure $4 \mathrm{~d}$ ). It is noteworthy that overexpression of TREM2 did not affect APP processing in the brain, as western blot analysis showed that the levels of full-length APP, $\beta$-CTFs, and $\alpha$-CTFs stayed unchanged after TREM2 overexpression (Figure 4e).

At 9 months of age, APPswe/PS1dE9 mice exhibited apparent inflammatory responses in the brain; mRNA levels 
a

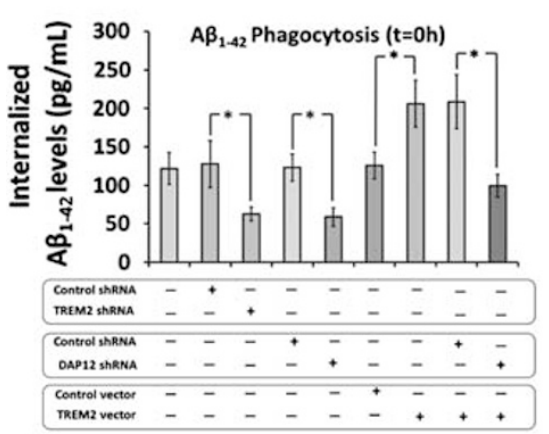

b

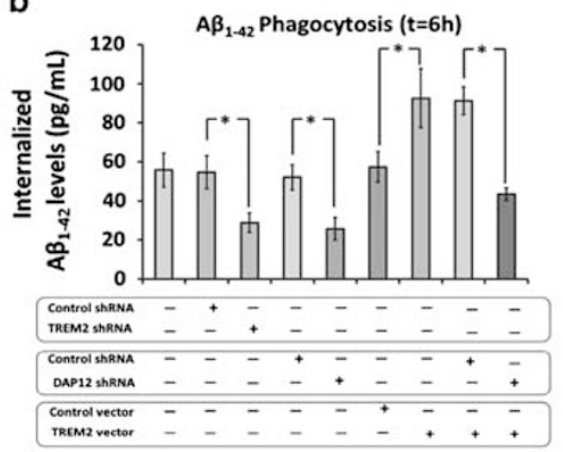

c

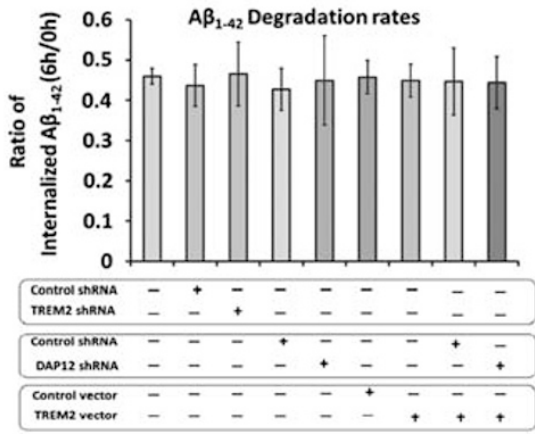

d

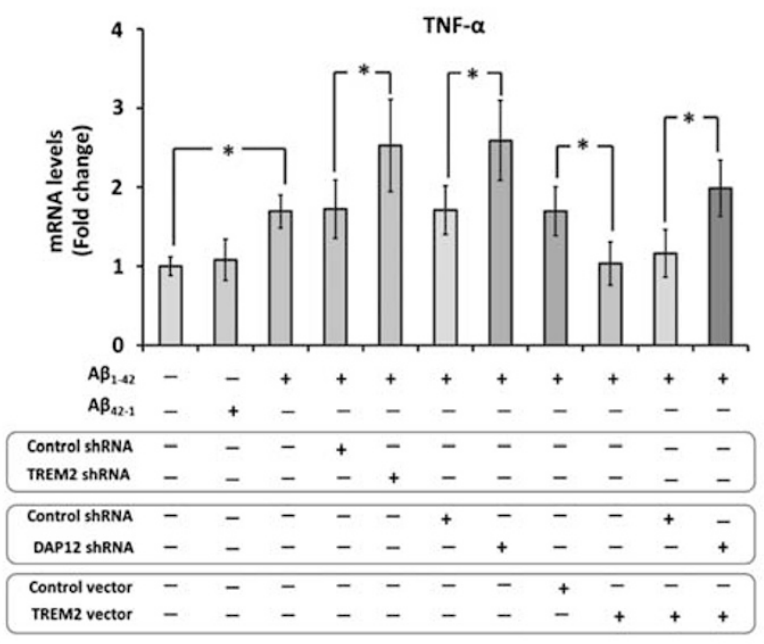

e
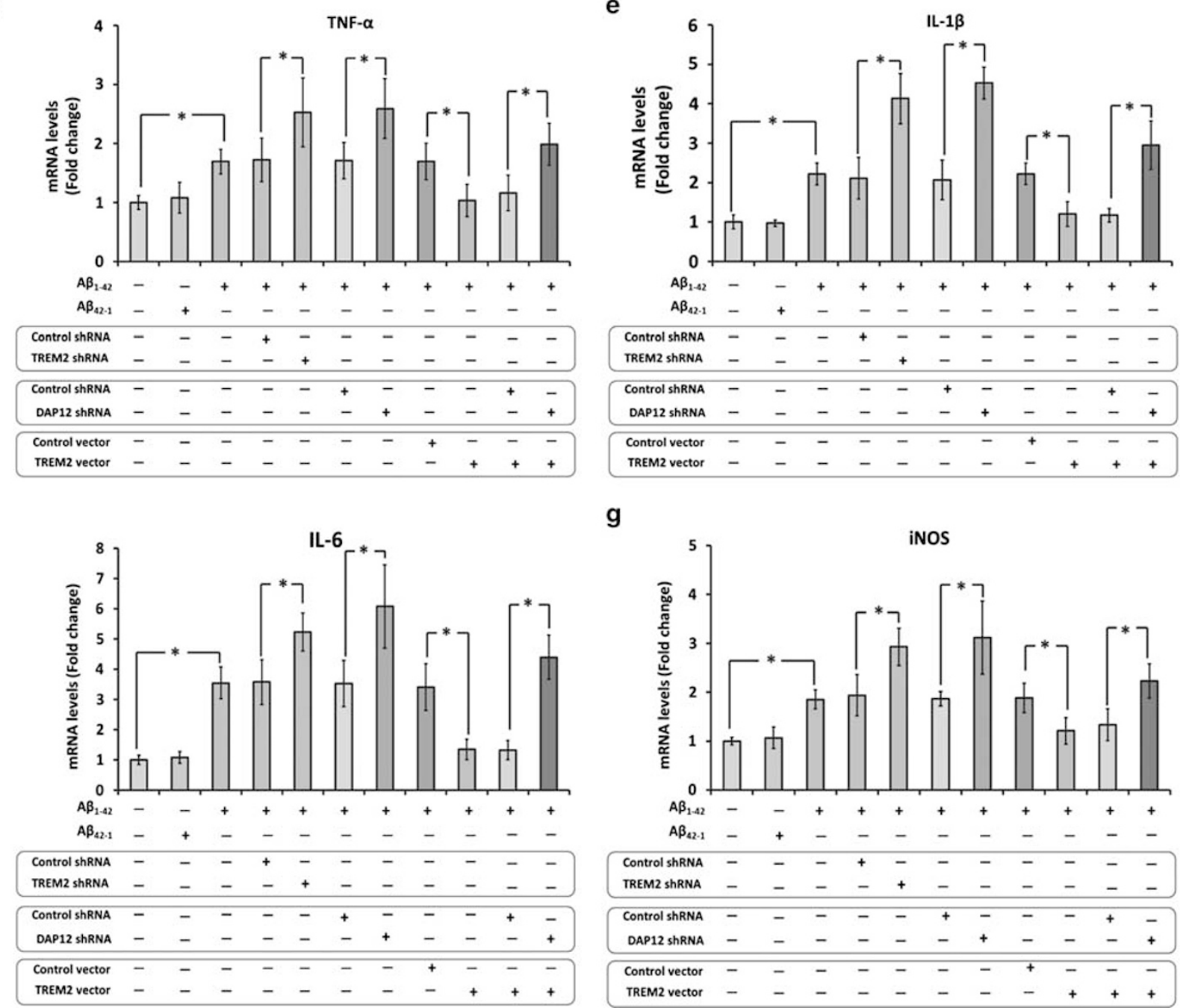

g

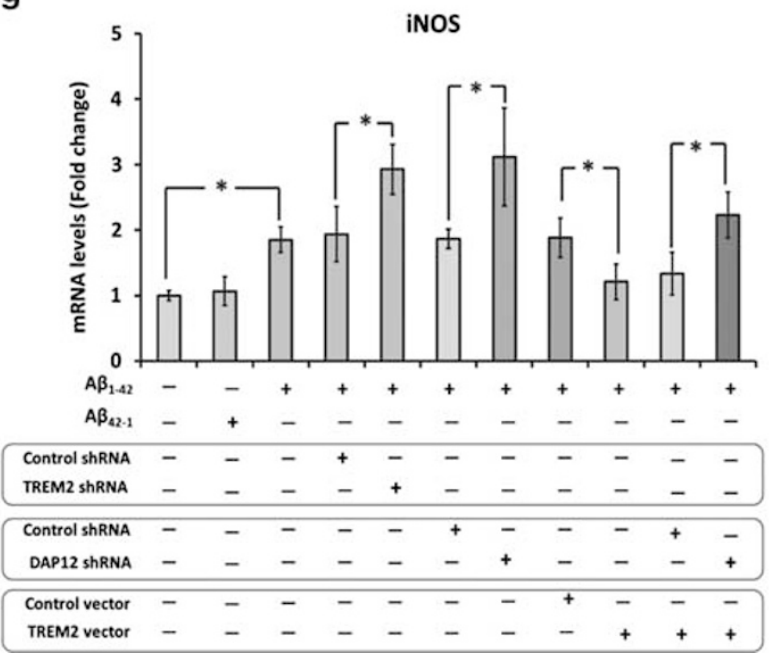

Figure 3 Triggering receptor expressed on myeloid cells 2 (TREM2) facilitates amyloid- $\beta(A \beta)_{\mid-42}$ phagocytosis and inhibits $A \beta_{\mid-42-\text { induced }}$ proinflammatory response in the primary microglia by a DAPI2-dependent manner. We first used a lentiviral strategy to manipulate TREM2 and DAPI 2 expression in the primary microglia. (a) To assess microglial $A \beta_{1-42}$ phagocytosis, the primary microglia was incubated with unlabeled $A \beta_{1-42}$ for $6 \mathrm{~h}$, and the intracellular $A \beta_{1-42}$ levels were measured by enzyme-linked immunoassay (ELISA). (b) Later, the $A \beta_{1-42}$ left in the medium was washed out, and the cells were maintained for an additional $6 \mathrm{~h}$ to allow for $A \beta_{1-42}$ degradation. The intracellular $A \beta_{1-42}$ levels were assessed by ELISA again. (c) The rate of $A \beta_{1-42}$ degradation was expressed as the ratio of remaining $A \beta_{1-42}(t=6 \mathrm{~h})$ to the total amount of internalized $A \beta_{42}(t=0 \mathrm{~h})$. Next, cells were stimulated with vehicle, $A \beta_{42-1}$, or $A \beta_{1-42}$ for $3 \mathrm{~h}$. (d-g) mRNA levels of tumor necrosis factor (TNF)- $\alpha$, interleukin (IL)-I $\beta$, IL-6, and inducible nitric oxide synthase (iNOS) within cells were measured by qRT-PCR. Data were analyzed by one-way analysis of variance (ANOVA) followed by Tukey's post hoc test. Columns represent mean \pm SEM. $* P<0.05$.

of proinflammatory cytokines including TNF- $\alpha \quad(n=8$, $q=7.35, P<0.05)$, IL-1 $\beta \quad(n=8, q=5.02, P<0.05)$, IL-6 $(n=8, q=12.27, \quad P<0.05)$, and iNOS $(n=8, q=5.92$,
$P<0.05)$ were significantly higher than those in the brain of WT mice (Figure $4 \mathrm{f}-\mathrm{i}$ ). As shown in Figure $4 \mathrm{f}-\mathrm{i}$, overexpression of TREM2 markedly reduced the expression 
of TNF- $\alpha$, IL- $1 \beta$, and IL- 6 by $39 \%(n=8, q=6.33, P<0.05)$, $31 \%(n=8, q=5.37, P<0.05)$, and $51 \%(n=8, q=10.81$, $P<0.05)$, respectively. Meanwhile, a $19 \%$ reduction of iNOS expression in the brain was observed after TREM2 overexpression, but this did not reach statistical significance (Figure $4 \mathrm{f}-\mathrm{i}, n=8, q=3.17$, NS).

As shown in Figure 5a-d, 9-month-old APPswe/PS1dE9 mice exhibited obvious neurodegenerative changes when compared with WT mice, as apparent neuronal (indicated by Nissl staining) and synaptic losses (indicated by synaptophysin levels) were observed in the brain. TREM2 overexpression markedly attenuated neuronal loss in the brain of APPswe/PS1dE9 mice, as the percentage of Nisslpositive neurons in the cortex and hippocampus was increased by $99 \%(n=8, q=14.11, P<0.05)$ and $102 \%$ $(n=8, q=17.65, P<0.05)$, respectively, after overexpression of TREM2 (Figures 5a-c). Meanwhile, overexpression of TREM2 also led to a $90 \%$ increase in synaptophysin levels $(n=8, q=7.17, P<0.05)$, implying a prevention of synaptic loss in APPswe/PS1dE9 mice brain (Figure 5d).

\section{TREM2 Overexpression Rescues Spatial Cognitive Impairment in APPswe/PS1dE9 Mice}

Finally, we examined the effects of TREM2 overexpression on spatial cognitive impairment in APPswe/PS1dE9 mice using Morris water maze test. First, the spatial learning abilities of mice were assessed by a 5-day hidden platform task. As indicated in Figure 5e, the WT mice performed significantly better than APPswe/PS1dE9 mice, suggesting APPswe/PS1dE9 mice displayed obvious deficiency in spatial learning ability at 9 months of age $(n=16$ per group; overall: $\mathrm{F}_{\text {genotype }}(1,150)=148.3, \quad P<0.05 ; \quad \mathrm{F}_{\text {days }}$ $(4,150)=111.7, \quad P<0.05 ; \quad \mathrm{F}_{\text {genotype } \times \text { days }} \quad(4,150)=5.42$, $P<0.05)$. APPswe/PS1dE9 mice that received TREM2 vector had a significantly better performance when compared with those that received control vector (Figure 5e; $n=16$ per group; overall: $\mathrm{F}_{\text {treatment }}(2,225)=36.59, P<0.05 ; \mathrm{F}_{\text {days }}$ $(4,225)=111.43, P<0.05 ; F_{\text {treatment }} \times$ days $\left.(8,225)=3.3, P<0.05\right)$, indicating that TREM2 overexpression significantly rescued spatial learning impairment. However, overexpression of TREM2 did not significantly affect spatial learning abilities in WT mice (Figure 5e; $n=16$ per group; overall: $F_{\text {treatment }}$ $(2,225)=0.56, \mathrm{NS} ; \mathrm{F}_{\text {days }(4,225)}=243.13, P<0.05 ; \mathrm{F}_{\text {treatment } \times \text { days }}$ $(8,225)=0.3$, NS). Next, to determine the effects of TREM2 overexpression on spatial memory, the platform was removed from the water and probe trials were conducted at $24 \mathrm{~h}$ after the last training trial. It should be noted that the data from probe trials are expressed as the percentage of time spent in target quadrant $v s$ the average time spent in other three quadrants. As shown in Figure 5f, APPswe/ PS1dE9 mice spent almost the same length of time in target quadrant than in any other quadrant $(n=16$ per group; $24.85 \pm 4.63 \%$ vs $25.01 \pm 1.61 \%$; $t=0.1331$, NS), whereas APPswe/PS1dE9 mice that received TREM2 vector spent significantly more time in target quadrant than in any other quadrant $(n=16$ per group; $35.76 \pm 7.55 \%$ vs $21.4 \pm 2.64 \%$; $t=7.816, \quad P<0.05)$. Overexpression of TREM2 did not significantly interfere with spatial memory in WT mice (Figure 5f). Of note, as indicated by an additional open-field activity test, TREM2 overexpression did not affect gross behavioral and motor functions (Supplementary Figure S5).
All these results suggested that TREM2 overexpression markedly rescued impairment of spatial memory in APPswe/PS1dE9 mice.

\section{DISCUSSION}

First, in the current study, we showed that TREM2 was only localized on microglia in the brain of APPswe/PS1dE9 mice, a transgenic model of $\mathrm{AD}$, confirming the previous observations from our group and others (Frank et al, 2008; Jiang et al, 2014a; Melchior et al, 2010). Meanwhile, TREM2 levels were found to be significantly elevated in the brain of APPswe/PS1dE9 mice in comparison with that of WT mice. This increase remained statistically significant when normalizing TREM2 levels to the expression of a microglial marker Iba-1, suggesting that the upregulation of TREM2 was attributed to the enhanced expression in individual microglia rather than the increase in total microglia numbers. This observation was in line with previous findings from Frank et al (2008), who showed that TREM2 expression was increased in amyloid plaqueassociated microglia in aged APP23 transgenic mice. More recently, by using TgCRND8 mice, another transgenic mouse model of AD, Guerreiro et al (2013) found that the expression of TREM2 was remarkably increased in microglia, providing more support to our findings. Interestingly, in the brain of APPswe/PS1dE9 mice, a positive correlation was observed between TREM2 expression and the levels of $\mathrm{A} \beta_{1-42}$, the most toxic $\mathrm{A} \beta$ species in $\mathrm{AD}$ pathogenesis (Yan and Wang, 2006), implying that the upregulation of TREM2 in microglia might be attributed to the increase in $\mathrm{A} \beta_{1-42}$ levels under AD context. To elucidate this causal relationship, we then stimulated primary microglia with $\mathrm{A} \beta_{1-42}$. For the first time, we showed that $\mathrm{A} \beta_{1-42}$ stimulation led to the upregulation of TREM2 in microglia. Meanwhile, this finding had been confirmed in vivo, as intracerebral injection of $\mathrm{A} \beta_{1-42}$ dose-dependently increased TREM2 levels in microglia in WT mice.

As the only cell expressing TREM2 in the brain, microglia can be activated by $\mathrm{A} \beta$ and can exert a dual function during AD progression (Hensley, 2010; Meda et al, 2001; Wyss-Coray, 2006). On the one hand, microglia participates in the clearance of $\mathrm{A} \beta$ deposition through its phagocytic activity. On the other hand, long-term stimulation with $\mathrm{A} \beta$ leads to the dysfunction of microglia, which is characterized by the overproduction of proinflammatory cytokines, subsequently leading to the bystander neuronal and synaptic damages. In this study, by manipulation of TREM2 expression in culture primary microglia, we showed that TREM2 facilitated microglial phagocytosis of $\mathrm{A} \beta_{1-42}$. This observation was supported by previous findings that TREM2 acted as a positive regulator of phagocytosis in microglia, as it could facilitate microglial phagocytosis of microsphere beads, apoptotic neurons, or $\mathrm{A} \beta_{1-40}$ in vitro (Hsieh et al, 2009; Melchior et al, 2010; Takahashi et al, 2005). Owing to the fact that TREM2 is a surface receptor of microglia, it is possible that TREM2 facilitates microglial $\mathrm{A} \beta_{1-42}$ phagocytosis by directly binding and internalizing this peptide. However, we cannot exclude the possibility that TREM2 facilitate $\mathrm{A} \beta_{1-42}$ phagocytosis by modulation of the function of other receptors participating in $\mathrm{A} \beta_{1-42}$ 


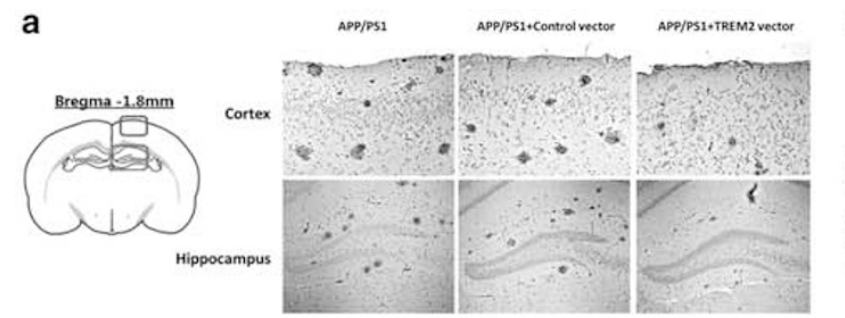
b

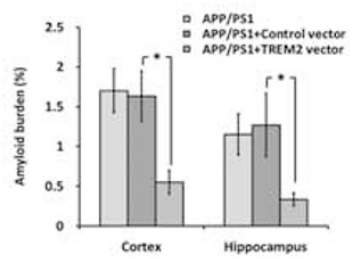

C
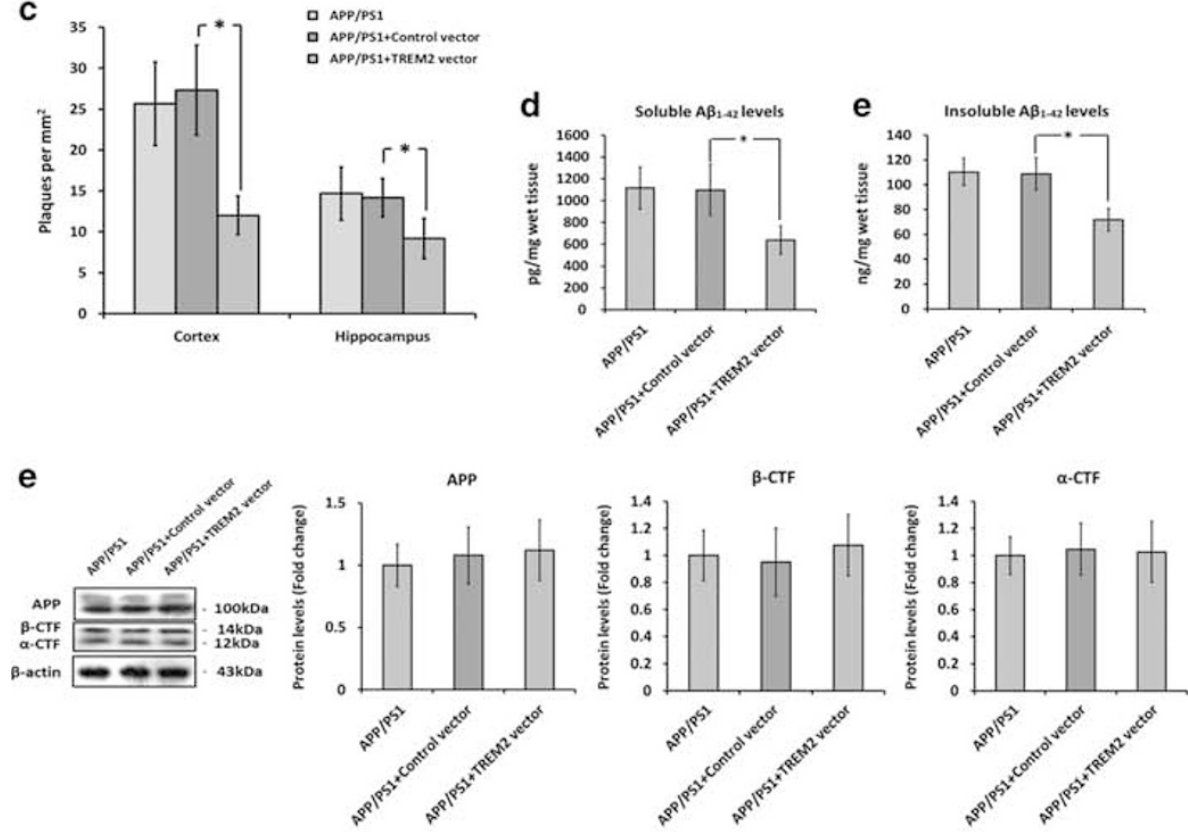

f

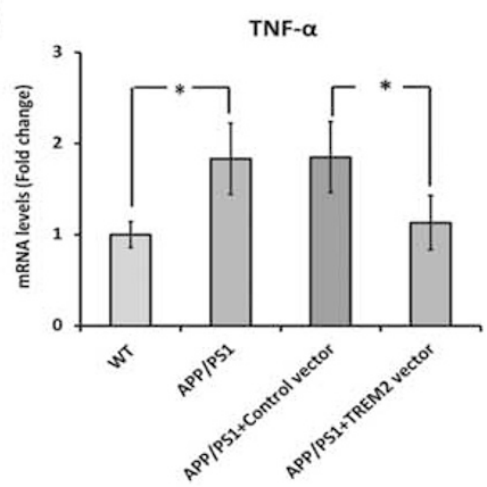

h

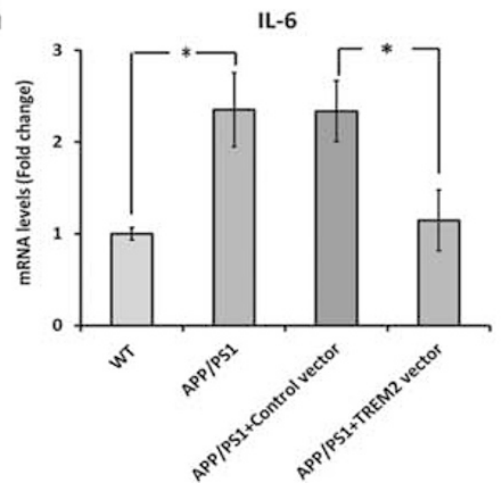

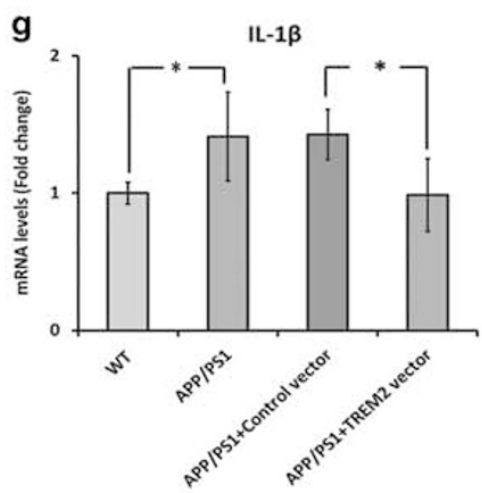

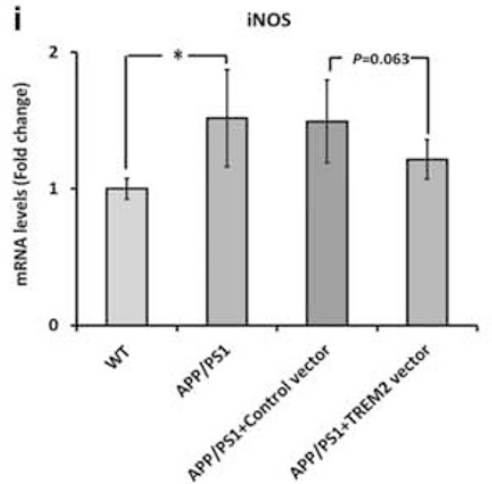


internalization, such as scavenger receptors and toll-like receptors (TLRs). In this study, we showed for the first time that TREM2 directly inhibited microglia-mediated proinflammatory responses after $\mathrm{A} \beta_{1-42}$ stimulation. This finding was supported by previous in vitro findings that TREM2 could inhibit microglial proinflammatory responses induced by LPS or apoptotic cells (Hsieh et al, 2009; Takahashi et al, 2005). However, the molecular mechanisms underlying this observation still remain unclear. In view of the fact that TLR had a major role in mediating $\mathrm{A} \beta$ triggered microglial proinflammatory responses (Jin et al, 2008; Liu et al, 2012), several lines of evidence suggested that TREM2 was a negative regulator of TLR signaling in myeloid cells (Hamerman et al, 2006; Ito and Hamerman, 2012; Turnbull et al, 2006); it is likely that the TREM2 attenuated $\mathrm{A} \beta_{1-42}$-triggered proinflammatory responses through inhibition of TLR signaling and functions. Moreover, we found that the modulation of TREM2 on microglial functions under $\mathrm{AD}$ context was dependent on it adaptor protein DAP12, as knockdown of DAP12 in microglia fully abolished the modulative effects of TREM2 on $\mathrm{A} \beta_{1-42}$ phagocytosis and $\mathrm{A} \beta_{1-42}$-induced proinflammatory responses. These observations were in line with previous findings that DAP12, the only signaling adaptor of TREM2, was required for the signaling and biologic functions of TREM2 in myeloid cells (Kiialainen et al, 2005; ParadowskaGorycka and Jurkowska, 2013). Interestingly, one of our preliminary experiments showed that DAP12 overexpression did not enhance TREM2-mediated microglial functions in vitro (data not shown), implying DAP12 might mediate the signaling of TREM2 in an 'all or none' manner under this condition. Taken together, all these data indicate that TREM2 could modulate microglial functions under $\mathrm{AD}$ context in a DAP12-dependent manner, and was likely to have a protective role against the progression of $\mathrm{AD}$.

To validate this hypothesis in vivo, we overexpressed TREM2 in the brain of APPswe/PS1dE9 mice. We found that overexpression of TREM 2 ameliorated $\mathrm{A} \beta$ deposition in the brain, as $\mathrm{A} \beta_{1-42}$ levels, as well as the amyloid plaques numbers and total amyloid burden, in the brain were markedly decreased after TREM2 overexpression. This effect was likely attributed to the enhanced microglial $\mathrm{A} \beta$ phagocytosis rather than attenuated $\mathrm{A} \beta$ production, as the levels of full-length APP and APP-CTFs in the brain were not altered by overexpression of TREM2. Meanwhile, we found that overexpression of TREM2 in the brain significantly attenuated neuroinflammation in the brain, as the production of several proinflammatory cytokines was significantly reduced after TREM2 overexpression. Coincidentally, a previous study by Piccio et al (2007)showed that TREM2 blockade exacerbated neuroinflammation in mice with experimental autoimmune encephalomyelitis. More direct evidence has, a recent study by Takahashi et al (2007), shown that intravenous application of TREM2transduced bone marrow-derived myeloid precursor cell markedly attenuated neuroinflammation and clinical symptoms in an animal model of multiple sclerosis (Takahashi et al, 2007). In combination with our in vitro data, the attenuation in neuroinflammation might be, at least partially, due to direct inhibition of TREM2 on microgliamediated proinflammatory response. However, as $\mathrm{A} \beta$ could lead to the overproduction of proinflammatory cytokines via activation of microglia, and therapies targeting $\mathrm{A} \beta$ could effectively ameliorate inflammatory responses in the brain (Meda et al, 2001; Wyss-Coray, 2006), it is also possible that TREM2 overexpression attenuated neuroinflammation via amelioration of $\mathrm{A} \beta$ neuropathology in this scenario. Furthermore, TREM2 overexpression in the brain of APPswe/PS1dE9 mice significantly attenuated neuronal and synaptic losses, which was accompanied by an improvement in spatial cognitive function. The neurotoxicity of $\mathrm{A} \beta$ and proinflammatory cytokines led to the neuronal and synaptic damage in cellular and animal models of AD (Calkins and Reddy, 2011; Gouras et al, 2010; Kapadia and Sakic, 2011), which could be effectively prevented by $\mathrm{A} \beta$-targeting therapy or anti-inflammatory treatment (Adolfsson et al, 2012; Varvel et al, 2009). On consideration of this evidence, the attenuation of neuronal and synaptic losses by TREM2 overexpression was likely attributed to the amelioration of $\mathrm{A} \beta$ neuropathology and neuroinflammation in this scenario. Meanwhile, as the neuronal and synaptic integrity was required for the maintenance of normal cognitive functions while neuronal and synaptic losses in brain was closely associated with cognitive deficits in AD (Burgess et al, 2002; Walsh and Selkoe, 2004), the improvement in spatial cognitive function induced by TREM2 overexpression could be a direct consequence of the attenuated neuronal and synaptic losses in brain. It is noteworthy that our results did not support a direct involvement of TREM2 in spatial cognitive functions, as overexpression of TREM2 in the brain of WT mice was not associated with an improvement of spatial learning and memory abilities in Morris water maze test. Collectively, these findings supported our hypothesis that TREM2

Figure 4 Triggering receptor expressed on myeloid cells 2 (TREM2) overexpression ameliorates amyloid- $\beta$ (A $\beta$ ) deposition and neuroinflammation in the brain of APPswe/PSI IE9 mice. First, a lentiviral strategy was used to overexpress TREM2 in the brain of APPswe/PSI dE9 mice. (a) Represent photos of amyloid plaques in the cortex and hippocampus of APPswe/PSI dE9 mice injected with vehicle, control vector or TREM2 vector. Amyloid plaques were detected by immunohistochemistry using an anti-A $\beta$ (4G8, which recognizes amino-acid residues 17-24 of A $\beta$ ) antibody. The plaques were visualized by a microscopy with $\times 200$ magnification; in the cortex: scale bar $=100 \mu \mathrm{m}$; in the hippocampus: scale bar $=200 \mu \mathrm{m}$. Blue box on the left atlas shows the region where the photo of the cortex was taken, and red box on the left atlas shows the region where the photo of the hippocampus was taken. (b) The amyloid burden in the cerebral cortex and hippocampus was expressed as the percentage of the area reactive with anti-A $\beta$ antibody (4G8) in relation to the total area analyzed. (c) The amyloid plaque density (number of plaques per $\mathrm{mm}^{2}$ ) was also calculated in the cerebral cortex and hippocampus. (d) The levels of soluble and insoluble A $\beta \mid-42$ in the brain were detected by enzyme-linked immunoassay (ELISA). (e) The protein levels of full-length APP, $\beta$-CTFs, and $\alpha$ CTFs in the brain were measured by western blot analysis. Data of western blot analysis were normalized to $\beta$-actin. It should be noted that cropped gels are used here, and the full-length gels are shown in Supplementary Figure S6. (f-i) mRNA levels of TNF- $\alpha$, IL-I $\beta$, IL-6, and iNOS in the brain of APPswe/PS I dE9 mice injected with vehicle, control vector, or TREM2 vector. All data were analyzed by one-way analysis of variance (ANOVA) followed by Tukey's post hoc test. Columns represent mean \pm SEM ( $n=8$ per group). $* P<0.05$. APP, amyloid precursor protein; $\beta$-CTFs, $C$-terminal fragment cleaved by $\beta$-secretase; $\alpha$-CTFs, C-terminal fragment cleaved by $\alpha$-secretase. 


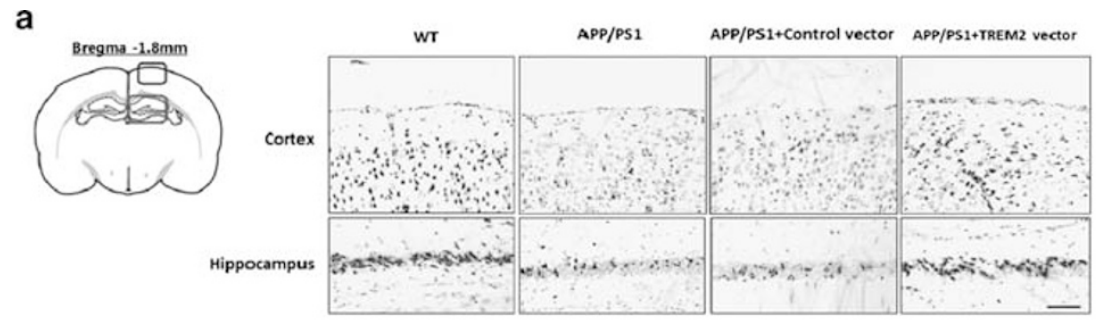

d

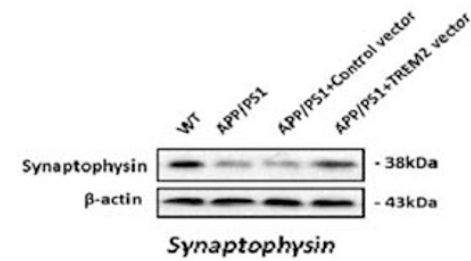

b

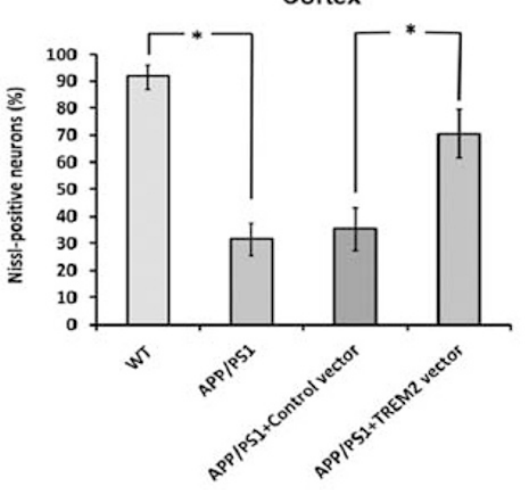

C

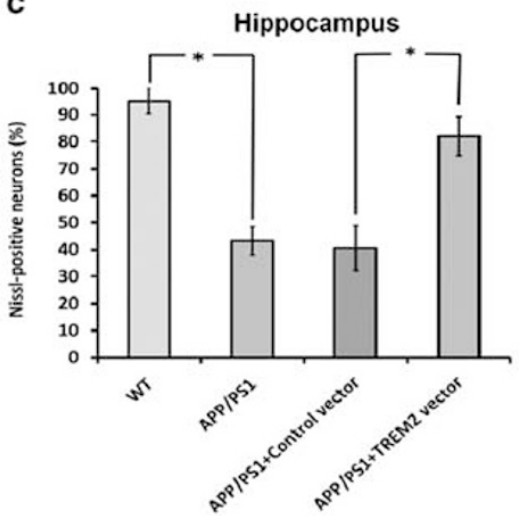

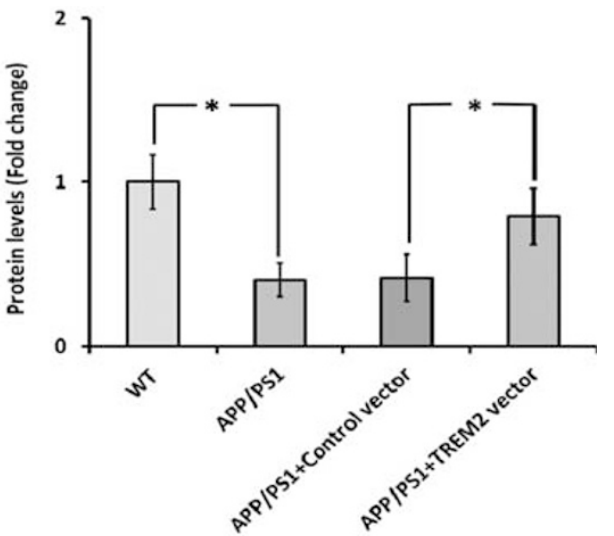

e

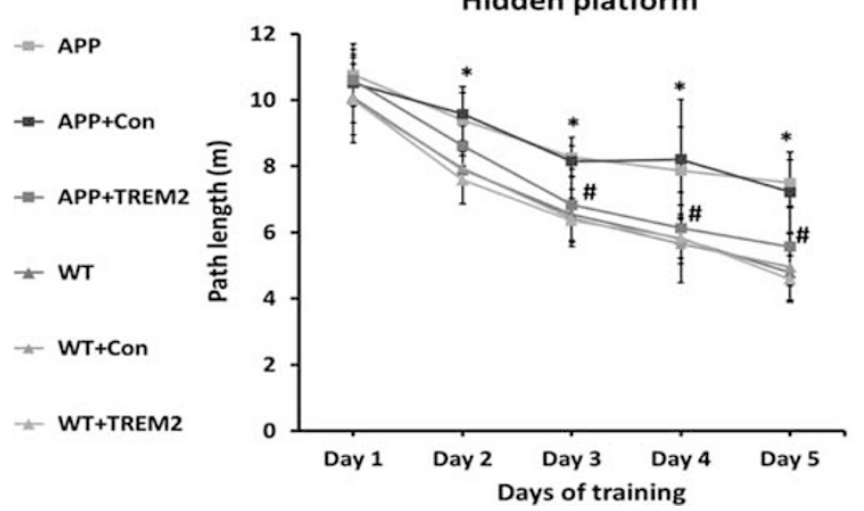

f

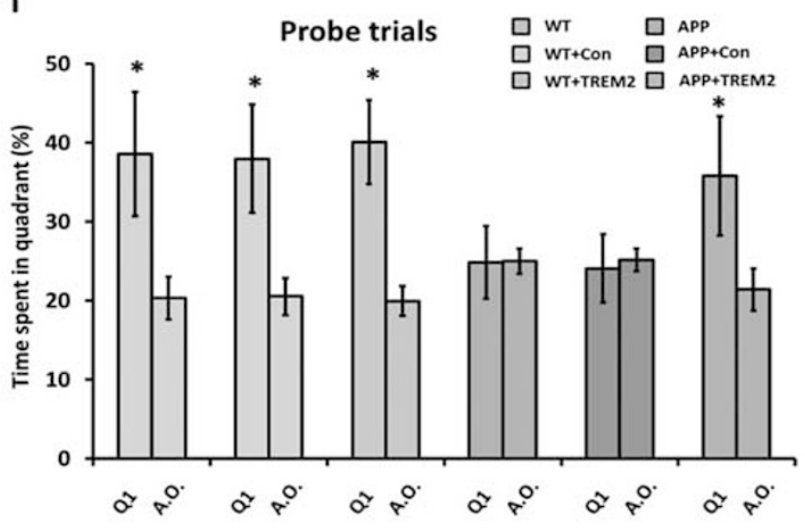

Figure 5 Triggering receptor expressed on myeloid cells 2 (TREM2) overexpression prevents neuronal and synaptic losses and rescues spatial cognitive deficits in APPswe/PSI dE9 mice. First, a lentiviral strategy was used to overexpress TREM2 in the brain of APPswe/PSIdE9 mice. (a) Nissl staining for detection of neuronal loss in the hippocampus and cortex of 9-month-old APPswe/PSI dE9 mice and wild-type (WT) mice. The neurons with dark violet nucleus and intact morphology were identified as Nissl-positive neurons. Scale bar $=100 \mu \mathrm{m}$. Blue box on the left atlas shows the region where the photo of the cortex was taken, and red box on the left atlas shows the region where the photo of the hippocampus was taken. (b and c) The APPswe/PSI dE9 mice showed obvious reduction in the percentage of Nissl-positive neurons in the hippocampus and cortex in comparison with WT mice, whereas this reduction was rescued by TREM2 overexpression. (d) Protein levels of synaptophysin in the brain of 9-month-old APPswe/PSI dE9 mice and WT mice were detected by western blot analysis. $\beta$-Actin was used as a loading control. It should be noted that cropped gels are used here, and the full-length gels are shown in Supplementary Figure S6. Data were analyzed by one-way analysis of variance (ANOVA) followed by Tukey's post hoc test. Columns represent mean \pm SEM ( $n=8$ per group). $* P<0.05$. Morris water maze test was conducted to assess the cognitive functions of animals at the last 6 days before mice were killed. The Morris water maze test consists of a hidden platform training at the last 5 consecutive days, plus a probe trial conducted at $24 \mathrm{~h}$ after the last hidden platform trial. (e) Path length of each group in the hidden platform training. Data were analyzed by two-way repeated-measures ANOVA followed by Tukey's post hoc test. ${ }^{*} P<0.05$ versus WT mice injected with vehicle. ${ }^{\#} P<0.05$ vs APPswe/PSI dE9 mice injected with control vector. (f) Data from probe trials are presented as the percentage of time spent in the target quadrant (QI) vs the averaged time spent in other three quadrants (a.o.). Data were analyzed by independent sample $t$-test. $* P<0.05$ vs averaged time spent in other three quadrants. Columns represent mean \pm SEM ( $n=16$ per group).

exerted a protective role in $\mathrm{AD}$ progression. Based on these findings, the upregulation of TREM2 under AD context can be interpreted as a compensatory mechanism in response to $\mathrm{A} \beta_{1-42}$ stimulation, which tries to couple with the $\mathrm{A} \beta$ deposition and neuroinflammation by modulation of microglial functions and subsequently arrests disease progression. However, this also raises many new questions that need to be answered, such as why this compensatory protective mechanism eventually fails.

It is noteworthy that the current study has some limitations. First, we used lentiviral strategy to overexpress TREM2 in the brain of APPswe/PS1dE9 mice. Although we showed, for the first time, that TREM2 overexpression protected against $\mathrm{AD}$ progression by modulation of 
microglia functions, this observation may not necessarily be robust owing to the individual variability in transduction efficiency. In our subsequent researches, triple transgenic mice overexpressing TREM2 in combination with APPswe/ PS1dE9 mutations will be generated to further validate this finding. Second, in contrast to our in vivo study showing the effects of TREM2 overexpression after a 2-month period, only short-term effects of TREM2 overexpression were reported by our in vitro experiment. In the future, additional long-term in vitro studies are highly warranted. Last, in this study we did not determine the underlying mechanisms by which $\mathrm{R} 47 \mathrm{H}$, a well-known variant located on exon 2 of TREM2, contributed to the etiology and pathogenesis of $\mathrm{AD}$, and future functional studies are needed to address this issue.

In conclusion, in the present study, we offer in vitro and in vivo evidence that the upregulation of TREM 2 serves as a compensatory response to $\mathrm{A} \beta_{1-42}$, which subsequently protects against AD progression by modulation of microglia functions. These findings provide insights into the role of TREM2 in AD pathogenesis, highlighting TREM2 as a potential therapeutic target for this disease.

\section{FUNDING AND DISCLOSURE}

The authors declare no conflict of interest.

\section{ACKNOWLEDGEMENTS}

This work was supported by the grants from the National Natural Science Foundation of China (to LT (81171209, $81371406)$ and JTY $(81000544,81471309)$ ), the grants from the Shandong Provincial Natural Science Foundation (to LT (ZR2011HZ001) and JTY (ZR2010HQ004)), the Medicine and Health Science Technology Development Project of Shandong Province (to LT (2011WSA02018) and JTY (2011WSA02020)), and the Innovation Project for Postgraduates of Jiangsu Province (to TJ (CXLX13_561)).

\section{REFERENCES}

Adolfsson O, Pihlgren M, Toni N, Varisco Y, Buccarello AL, Antoniello $\mathrm{K}$ et al (2012). An effector-reduced anti-beta-amyloid (Abeta) antibody with unique abeta binding properties promotes neuroprotection and glial engulfment of Abeta. J Neurosci 32: 9677-9689.

Burgess N, Maguire EA, O'Keefe J (2002). The human hippocampus and spatial and episodic memory. Neuron 35: 625-641.

Calkins MJ, Reddy PH (2011). Amyloid beta impairs mitochondrial anterograde transport and degenerates synapses in Alzheimer's disease neurons. Biochim Biophys Acta 1812: 507-513.

Colonna M (2003). TREMs in the immune system and beyond. Nate Rev Immunol 3: 445-453.

Daws MR, Lanier LL, Seaman WE, Ryan JC (2001). Cloning and characterization of a novel mouse myeloid DAP12-associated receptor family. Eur J Immunol 31: 783-791.

Dodart JC, Marr RA, Koistinaho M, Gregersen BM, Malkani S, Verma IM et al (2005). Gene delivery of human apolipoprotein E alters brain Abeta burden in a mouse model of Alzheimer's disease. Proc Natl Acad Sci USA 102: 1211-1216.

Frank S, Burbach GJ, Bonin M, Walter M, Streit W, Bechmann I et al (2008). TREM2 is upregulated in amyloid plaque-associated microglia in aged APP23 transgenic mice. Glia 56: 1438-1447.
Gouras GK, Tampellini D, Takahashi RH, Capetillo-Zarate E (2010). Intraneuronal beta-amyloid accumulation and synapse pathology in Alzheimer's disease. Acta Neuropathol 119: 523-541.

Griciuc A, Serrano-Pozo A, Parrado AR, Lesinski AN, Asselin CN, Mullin $\mathrm{K}$ et al (2013). Alzheimer's disease risk gene CD33 inhibits microglial uptake of amyloid beta. Neuron 78: 631-643.

Guerreiro R, Wojtas A, Bras J, Carrasquillo M, Rogaeva E, Majounie E et al (2013). TREM2 variants in Alzheimer's disease. $N$ Engl J Med 368: 117-127.

Hamerman JA, Jarjoura JR, Humphrey MB, Nakamura MC, Seaman WE, Lanier LL (2006). Cutting edge: inhibition of TLR and $\mathrm{FcR}$ responses in macrophages by triggering receptor expressed on myeloid cells (TREM)-2 and DAP12. J Immunol 177: 2051-2055.

Hensley K (2010). Neuroinflammation in Alzheimer's disease: mechanisms, pathologic consequences, and potential for therapeutic manipulation. J Alzheimer's Dis 21: 1-14.

Hsieh CL, Koike M, Spusta SC, Niemi EC, Yenari M, Nakamura MC et al (2009). A role for TREM2 ligands in the phagocytosis of apoptotic neuronal cells by microglia. J Neurochem 109: $1144-1156$.

Ito H, Hamerman JA (2012). TREM-2, triggering receptor expressed on myeloid cell-2, negatively regulates TLR responses in dendritic cells. Eur J Immunol 42: 176-185.

Jiang T, Yu JT, Zhu XC, Tan L (2013). TREM2 in Alzheimer's disease. Mol Neurobiol 48: 180-185.

Jiang T, Yu JT, Zhu XC, Tan MS, Gu LZ, Zhang YD et al (2014a). Triggering receptor expressed on myeloid cells 2 knockdown exacerbates aging-related neuroinflammation and cognitive deficiency in senescence-accelerated mouse prone 8 mice. Neurobiol Aging 35: 1243-1251.

Jiang T, Yu JT, Zhu XC, Tan MS, Wang HF, Cao L et al (2014b). Temsirolimus promotes autophagic clearance of amyloid-beta and provides protective effects in cellular and animal models of Alzheimer's disease. Pharmacol Res 81: 54-63.

Jin JJ, Kim HD, Maxwell JA, Li L, Fukuchi K (2008). Toll-like receptor 4-dependent upregulation of cytokines in a transgenic mouse model of Alzheimer's disease. J Neuroinflamm 5: 23.

Jonsson T, Stefansson H, Steinberg S, Jonsdottir I, Jonsson PV, Snaedal J et al (2013). Variant of TREM2 associated with the risk of Alzheimer's disease. N Engl J Med 368: 107-116.

Kapadia M, Sakic B (2011). Autoimmune and inflammatory mechanisms of CNS damage. Progr Neurobiol 95: 301-333.

Kiialainen A, Hovanes K, Paloneva J, Kopra O, Peltonen L (2005). Dap12 and Trem2, molecules involved in innate immunity and neurodegeneration, are co-expressed in the CNS. Neurobiol Dis 18: $314-322$.

Larson ME, Lesne SE (2012). Soluble Abeta oligomer production and toxicity. J Neurochem 120(Suppl 1): 125-139.

Liu S, Liu Y, Hao W, Wolf L, Kiliaan AJ, Penke B et al (2012). TLR2 is a primary receptor for Alzheimer's amyloid beta peptide to trigger neuroinflammatory activation. I Immunol 188: 1098-1107.

Meda L, Baron P, Scarlato G (2001). Glial activation in Alzheimer's disease: the role of Abeta and its associated proteins. Neurobiol Aging 22: 885-893.

Melchior B, Garcia AE, Hsiung BK, Lo KM, Doose JM, Thrash JC et al (2010). Dual induction of TREM2 and tolerance-related transcript, Tmem176b, in amyloid transgenic mice: implications for vaccine-based therapies for Alzheimer's disease. ASN Neuro 2: e00037.

Moreno-Gonzalez I, Estrada LD, Sanchez-Mejias E, Soto C (2013). Smoking exacerbates amyloid pathology in a mouse model of Alzheimer's disease. Nat Commun 4: 1495.

Neumann H, Daly MJ (2013). Variant TREM2 as risk factor for Alzheimer's disease. N Engl J Med 368: 182-184. 
Neumann H, Takahashi K (2007). Essential role of the microglial triggering receptor expressed on myeloid cells-2 (TREM2) for central nervous tissue immune homeostasis. J Neuroimmunol 184: 92-99.

Paloneva J, Manninen T, Christman G, Hovanes K, Mandelin J, Adolfsson $\mathrm{R}$ et al (2002). Mutations in two genes encoding different subunits of a receptor signaling complex result in an identical disease phenotype. Am J Hum Genet 71: 656-662.

Paradowska-Gorycka A, Jurkowska M (2013). Structure, expression pattern and biological activity of molecular complex TREM2/DAP12. Hum Immunol 74: 730-737.

Piccio L, Buonsanti C, Mariani M, Cella M, Gilfillan S, Cross AH et al (2007). Blockade of TREM-2 exacerbates experimental autoimmune encephalomyelitis. Eur JImmunol 37: 1290-1301.

Takahashi K, Prinz M, Stagi M, Chechneva O, Neumann H (2007). TREM2-transduced myeloid precursors mediate nervous tissue debris clearance and facilitate recovery in an animal model of multiple sclerosis. PLoS Med 4: e124.

Takahashi K, Rochford CD, Neumann H (2005). Clearance of apoptotic neurons without inflammation by microglial trigger- ing receptor expressed on myeloid cells-2. J Exp Med 201: 647-657.

Tan MS, Yu JT, Jiang T, Zhu XC, Guan HS, Tan L (2014). IL12/23 p40 inhibition ameliorates Alzheimer's disease-associated neuropathology and spatial memory in SAMP8 mice. J Alzheimer's Dis 38: 633-646.

Turnbull IR, Gilfillan S, Cella M, Aoshi T, Miller M, Piccio L et al (2006). Cutting edge: TREM-2 attenuates macrophage activation. J Immunol 177: 3520-3524.

Varvel NH, Bhaskar K, Kounnas MZ, Wagner SL, Yang Y, Lamb BT et al (2009). NSAIDs prevent, but do not reverse, neuronal cell cycle reentry in a mouse model of Alzheimer disease. J Clin Invest 119: 3692-3702.

Walsh DM, Selkoe DJ (2004). Deciphering the molecular basis of memory failure in Alzheimer's disease. Neuron 44: 181-193.

Wyss-Coray T (2006). Inflammation in Alzheimer disease: driving force, bystander or beneficial response? Nat Med 12: 1005-1015.

Yan Y, Wang C (2006). Abeta42 is more rigid than Abeta40 at the C terminus: implications for Abeta aggregation and toxicity. $\mathrm{J} \mathrm{Mol}$ Biol 364: 853-862.

Supplementary Information accompanies the paper on the Neuropsychopharmacology website (http://www.nature.com/npp) 